\title{
IDENTIFICATION FOR ION-BASED FERTIGATION CONTROL IN SOILLESS GREENHOUSE CULTIVATION
}

\author{
G. van Straten ${ }^{1}$, G.J. van Dijk ${ }^{1}$, R.J.C. van Ooteghem ${ }^{1}$, \\ Th. H. Gieling ${ }^{2}$, H.J.J. Janssen ${ }^{2}$, W.F. Mulckhuijse ${ }^{3}$ \\ ${ }^{I}$ Systems and Control Group, Wageningen University, Bornsesteeg 59, \\ 6708 PD Wageningen, The Netherlands, \\ e-mail: gerrit.vanstraten@wur.nl (corresponding author) \\ ${ }^{2}$ Agrotechnology and Food Innovations BV, PO Box 17, \\ 6700 AA Wageningen, The Netherlands \\ ${ }^{3}$ Hydrion BV, PO Box 522, 6700 AM. Wageningen, The Netherlands
}

\begin{abstract}
Progress in instrumentation and the need for quality control stimulate the rethinking of irrigation and fertilisation (fertigation) of crops in greenhouses. Feedback control using ion specific electrodes in the drain offers opportunities for automatic demand satisfaction. The fertigation system is characterised by pulse-wise water supply, which puts unconventional challenges to the measurement system. Limitations from practice require identification and control design procedures to be robust and simple. This paper introduces a reduced model for the system and describes the practical identification of the nutrient dynamics in a measurement gully on a time scale that is relevant to the crop. Copyright (C) 2005 IFAC
\end{abstract}

Keywords: greenhouses, fertigation, feedback control, plant nutrition, identification

\section{INTRODUCTION}

Current fertigation practice in soil-less greenhouse crop cultivation is essentially open loop in nature. Water is supplied in pulses in proportion to the solar radiation on the basis of experience about the water need. The irrigation water is prepared according to a fixed recipe, while $\mathrm{EC}$ and $\mathrm{pH}$ are adjusted by automatic feedback control, without feedback from the root environment of the crop. In view of environmental protection and efficient water use, water is being recycled, which complicates the preparation of feed solutions. Moreover, due to the desire for better control of crop quality, there is a need for more control over the actual root environment. The introduction of ion-specific sensors and analysers allows the introduction of feedback control to better suit the needs of the grower. The Hydrion-line project is a cooperative project of commercial firms, research institutes and university to arrive at new commercial controllers to this end.

\section{CRITERIA FOR FERTIGATION CONTROLLER DESIGN}

The primary purpose of the irrigation system is to provide water and nutrients in such a way that these resources do not limit crop growth and development. The uptake of water and important macro-ions is regulated by the plant itself. Above a certain threshold the uptake is practically independent on water potential and nutrient concentration in the root zone. The threshold is determined by kinetic and 


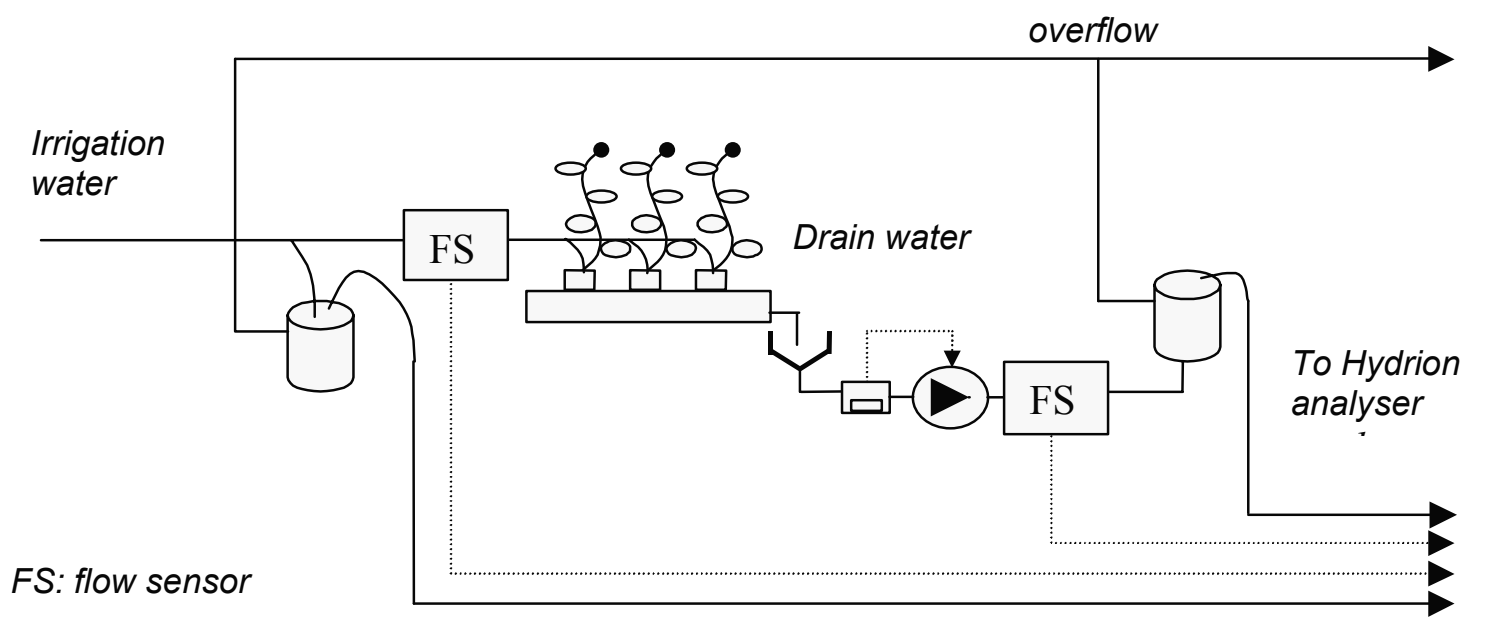

Fig. 1. System layout of the measurement gully.

transport considerations (LeBot et al., 1998, van Straten and Gieling, 2004). On the other hand, if there is too much water, damage may occur due to oxygen depletion in the root zone. Also, at high total ion concentrations (EC), water stress may occur as it becomes more difficult for the plant to overcome osmotic pressure, at the expense of yield, but to the benefit of fruit mineral content and dry weight accumulation (Dorais et al., 2001). Generally, it is assumed that there exists an operating range for water and nutrients that serves the need of the plants in the best possible way. So, it is desirable to create a control system that is able to keep the substrate water content and individual ion concentrations in the operating range at all times, thus automatically compensating for the uptake by the crop, while allowing control over EC.

In commercial greenhouses, water is supplied in irrigation cycles. This is done for technical and cost reasons, but it is also favourable for the crop, as it stimulates root development. So, there are fluctuations in water content and concentration during the fertigation cycle, which does not harm as long as the conditions remain within the operating range. However, the pulse-wise operation constitutes some difficulties from the point of view of data collection and identification. This paper describes the results of identification experiments performed for the design of an ion-specific controller. Since the controller will be a commercial product, emphasis is put on relatively simple, yet reliable and confident procedures.

\section{MATERIALS AND METHODS}

\subsection{Measurement gully and irrigation system}

The overall system layout in all experiments is according to figure 1 . A representative measurement gully consisting of two sections has been used. Each section contained 8 plants. The 16 plants were placed in rockwool pots (volume together 12 1), which in turn have been placed on top of a rockwool substrate mat (Grodan, type Expert, total slab volume 1201 , saturation retention about $80 \%$ ). The mat as a whole is wrapped in white plastic in order to prevent evaporation from the substrate. Drainage slits have been made to allow drain water to leave the substrate. A first set of experiments was done with tomato, a second set with cucumber.

Each plant is irrigated by its own dripper. The irrigation water is prepared by a nutrient dosage unit (Hortimax Modifeed). Liquid fertiliser solutions from an array of 8 stock concentrates are injected in a dispenser tube, and mixed in a mixing reservoir until the required concentrations have been reached, before being released to the greenhouse.

Because of the electro-neutrality condition and the wish to avoid precipitates, the desired ion composition is not entirely free (de Rijck and Schrevens, 1995). EC and $\mathrm{pH}$ are controlled independently. The drain of the two sections is collected in a single collector bin, so that the gullies are effectively in parallel. The flow rate of the ingoing and outgoing flow is monitored with a turbine flow meter.

\subsection{Ion-specific measurements and data acquisition}

A measuring unit based upon ion-selective electrodes (Hydrion) measures concentrations of individual ions both in irrigation as well as drain water. The measurable ions and concentrations are $\mathrm{HCO}_{3}{ }^{-}, \mathrm{CO}_{2}$, $\mathrm{NO}_{3}^{-}, \mathrm{Cl}^{-}, \mathrm{K}^{+}, \mathrm{Ca}^{2+}, \mathrm{Na}^{+}, \mathrm{NH}_{4}^{+}$and $\mathrm{pH}$. In addition, $\mathrm{EC}$ is measured in both streams. The Hydrion analyser consists of a set of ion-specific electrodes, an internal liquid transport system, and embedded 
software to handle temperature compensation, autocalibration, A/D conversion and error detection. A calibration cycle takes about 7 minutes. Measurement of irrigation and drain are done with the same equipment, which means that it may take up to 15 minutes before a measurement becomes available. The measuring unit requires about $250 \mathrm{ml}$ of sample, which sets a lower limit to the sampling interval. Sampling is triggered by counts of a cumulative flow sensor indicating sufficient water. All measurement data are directly stored in a real time database for on line process control and convenient remote data retrieval.

\subsection{Drain flow control}

The feedback principle also applies to the water dosage. A system that keeps the drain flow constant by manipulating the irrigation automatically compensates for the water uptake demand of the crop (Gieling et al., 2000). The instantaneous drain flow cannot be kept constant, because the irrigation is given in pulses. Instead, in the experiments, the time averaged drain flow was kept constant by varying the interval time between the irrigation pulses.

\subsection{Data processing}

A typical flow pattern of the drain is shown in Figure 2. Flow rate was deduced from cumulative flow recorded at 1 minute intervals, for both irrigation water and drain water.

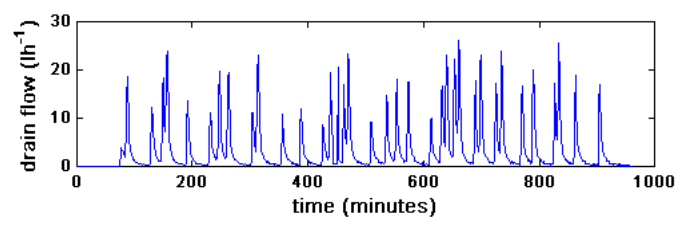

Fig. 2. Typical drain flow pattern.

Since the nutrient sampler needs a sufficient amount of water, its sampling time is not equidistant. Moreover, in periods without sufficient drain, no measurements are available. In the data processing the status information provided by the analyser is used to remove invalid data. Data for which it was known that there were other technical problems were also removed. Sometimes unexpected large jumps occur, which are physically impossible. Therefore, the raw data were filtered in such a way that large jumps were ironed out by allowing a maximum slope up or down from the previous valid measurement.

In this way a signal is obtained that is suitable for analysis. It is clear that no information can be retrieved for the behaviour within a pulse.

\section{SYSTEM IDENTIFICATION EXPERIMENTS}

\subsection{Equivalence between EC and dissolved ions}

A preliminary experiment was performed by studying the step response of $\mathrm{NO}_{3}{ }^{-}, \mathrm{NH}_{4}{ }^{+}, \mathrm{Ca}^{2+}, \mathrm{K}^{+}$ and $\mathrm{EC}$ in a gully with tomato plants. Only a step up was given. The second order grey-box model described in section 6 was used to represent the data. The results showed that parameters obtained on the EC data could be used without modification to describe the behaviour of $\mathrm{K}^{+}$and $\mathrm{NO}_{3}^{-}$, except that the ion uptake is different. This finding confirms that dissolved ions behave in the same fashion, and hence can be controlled with identical controllers. Results for $\mathrm{Ca}^{2+}$ and $\mathrm{NH}_{4}^{+}$were not decisive, due to difficulties in obtaining reliable data. In the case of $\mathrm{Ca}^{2+}$ this is due to the lower sensitivity (bi-valent ion) in conjunction with problems with consistency of the electrode membrane. The $\mathrm{NH}_{4}^{+}$analysis is hindered by $\mathrm{K}^{+}$, and since there is much more $\mathrm{K}^{+}$in greenhouse recycle water, no accurate measurement can be obtained.

\subsection{Step response tests}

Next, experiments were performed with a new measurement gully and cucumber plants. Analysis of the data showed that good fits could be obtained using a first order with delay model. In order to obtain the static gain this result was obtained on a mass-to-mass basis, using the cumulative masses in and out as signals. As expected, the static gain in this way is always less than 1 . However, the cumulative data are less suitable to obtain reliable estimates of the time constant and dead time. Since the plant uptake is going to be treated as disturbance, it was decided to obtain the nutrient dynamics from experiments without plants first.

Hence, a third experiment was performed, without plants. After an initialisation period, the setting for the $\mathrm{EC}$ in the irrigation water was increased from 2 to $4 \mathrm{mS} \mathrm{cm}$, by modifying the individual ion concentration settings in the recipe (step up). After 6 days, the settings returned to $2 \mathrm{mS} \mathrm{cm}^{-1}$ (step down) for 7 days. The drainage flow was set at $1 \mathrm{lh}^{-1}$. Finally, a step up experiment was made with a drain flow of $31 \mathrm{~h}^{-1}$. It should be noted that the actual concentrations realised in the irrigation water deviate from these ideal patterns. This is due to the irrigation unit, where it takes time to produce the liquid in the desired composition, and where it is sometimes impossible to exactly produce the required recipe. In all cases, the actually measured time trajectories were used as input to the model, using linear interpolation during the inter-sample intervals. 


\section{RESULTS}

\subsection{Fit results}

A first order model with input delay is used to describe the data. The static gain $(\mathrm{K})$, the time constant $(\tau)$ and the dead time $t_{\mathrm{d}}$ are the parameters $p$ to be estimated. It appears that the estimates are highly correlated. Since there are no plants, the static gain is expected to be equal to 1 . Therefore, $\mathrm{K}$ was set to 1 , which clearly improved the consistency of the results. In table 1, the quality of the fit is reported as

$$
F_{o}=100\left(1-\frac{\left\|y_{\text {data }}-y_{\text {sim }}\right\|_{2}}{\left\|y_{\text {data }}-\bar{y}_{\text {data }}\right\|_{2}}\right)
$$

At a perfect fit $F_{o}=100$. In addition, assuming white residual error, the correlation and uncertainty of the parameters is computed as

$$
\operatorname{cov}(\hat{p})=\frac{s s q}{N-n_{p}}\left(J^{T} J\right)^{-1}
$$

where $s s q$ is the residual sum of squares, $N$ the number of data points, $n_{p}$ the number of parameters $p$, and $J$ is the Jacobian matrix with elements

$$
J_{i j}=\left.\frac{\partial y_{i}}{\partial p_{j}}\right|_{\hat{p}}
$$

The coefficient of variation in $\%$ as reported in table 1 is

\begin{tabular}{|c|c|c|c|c|}
\hline dataset & ion & $\tau$ & $t_{d}$ & $F_{o}$ \\
\hline \multirow{5}{*}{ Up } & EC & 5558 & 70 & 81 \\
\hline & $\mathrm{cv} \%$ & 1 & 64 & \\
\hline & $\mathrm{K}^{+}$ & 4830 & 147 & 72 \\
\hline & $\mathrm{cv} \%$ & 12 & 236 & \\
\hline & $\mathrm{NO}_{3}^{-}$ & 5231 & 62 & 80 \\
\hline \multirow{6}{*}{ Down } & $\mathrm{EC}$ & $9092^{13}$ & $\begin{array}{l}652 \\
0\end{array}$ & 33 \\
\hline & $\mathrm{cv} \%$ & 10 & high & \\
\hline & $\mathrm{K}^{+}$ & 6591 & 0 & 25 \\
\hline & $\mathrm{cv} \%$ & 221 & high & \\
\hline & $\mathrm{NO}_{3}^{-}$ & 8953 & 0 & 7 \\
\hline & $\mathrm{cv} \%$ & 79 & high & \\
\hline \multirow[t]{5}{*}{ Up $31 \mathrm{~h}^{-1}$} & & 1947 & 42 & 24 \\
\hline & cv\% & 18 & 303 & \\
\hline & $\mathrm{K}^{+}$ & 2101 & 0 & 6 \\
\hline & $\mathrm{cv} \%$ & 87 & high & \\
\hline & $\mathrm{NO}_{3}^{-}$ & $\begin{array}{c}1792 \\
112\end{array}$ & $\begin{array}{c}0 \\
\text { high }\end{array}$ & 19 \\
\hline
\end{tabular}

$$
c v(p)=100 \frac{\sigma_{p}}{p}, \quad \text { with } \sigma=\sqrt{\operatorname{diag}(\operatorname{cov}(\hat{p}))}
$$

Table 1: Fit results $(\mathrm{K}=1$, times in minutes)

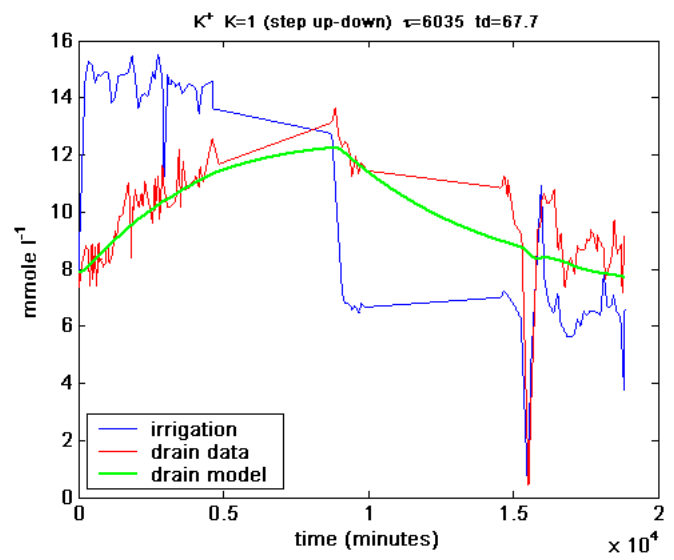

Fig. 3. Fit on potassium concentration data.

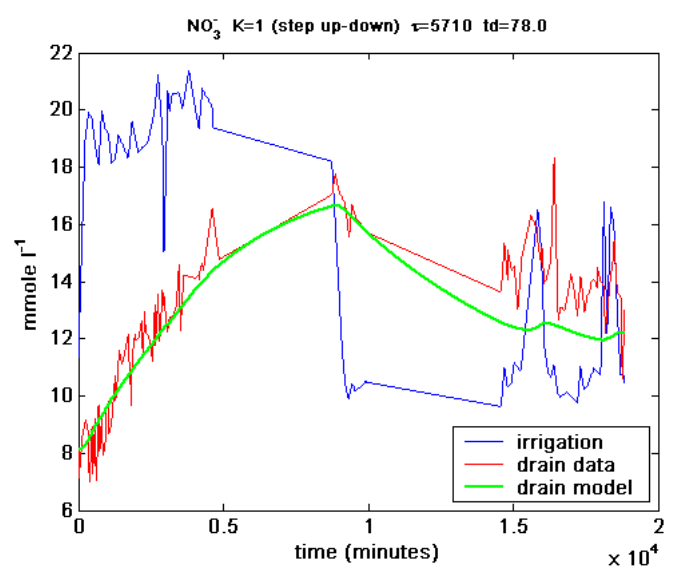

Fig. 4. Fit on nitrate concentration data.

The first step up experiment yields the best model fits (see Figures 3 and 4). Fixing $\mathrm{K}$ to 1 improves the accuracy of the remaining parameters, with little effect on the residual sum of squares (not shown).

The dead time estimate has a large estimation uncertainty. It is also strongly negatively correlated with $\tau$. Thus a good policy would be to fix both $\mathrm{K}$ (known from physics) and $t_{d}$ (too uncertain), and estimate just $\tau$. Fixing $\mathrm{K}$ also improves the consistency among the various ions of dissolved components, which, theoretically, should have the same time constants. In the step up experiment, the time constant is about 5500 minutes, which compares nicely with the physically expected value as will be shown in section 6.2.

The experiment with $31 \mathrm{~h}^{-1}$ should lead to time constants that are roughly 3 times as short. Since the experiment lasted only a few days, accurate estimation of $\mathrm{K}$ is not possible. However, despite the short period, the data obtained with $\mathrm{K}$ set to 1 indicate a time constant of 1800-2000 minutes, which is 2.5-3 times as small as with the original flow rate. 


\subsection{Cross-validation}

The step-up / step-down data were also used to cross validate the model. Parameters obtained from the step up and those of the step down measurements were used to simulate the complete sequence. In addition, the expected physical parameters, obtained from the geometrics of the measurement gully together with an estimate of the water retention in the substrate mat, were used.

The results are shown in figure 5. Indeed, there is some indication that the downward step has a somewhat larger time constant.

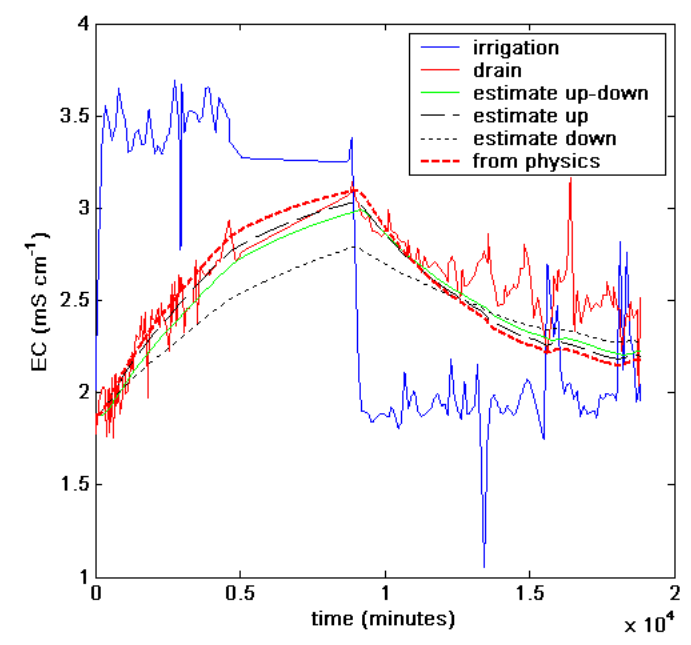

Fig. 5 Cross validation on EC.

\section{TWO-COMPARTMENT MODEL}

\subsection{Analysis}

The ion-concentration in the substrate mat is nonuniform. It is therefore necessary to address the question whether success can be expected from a controller based on regulating drain concentrations (Kläring, 2001). Heinen (2001) developed a 2-D model that can be used to analyse the dynamics of the distribution in the substrate. Here, a reduced model approach is used to clarify the main issues.

Consider the following nutrient compartmental model

$$
\begin{aligned}
& \varepsilon V_{1} \frac{d C_{1}}{d t}=F_{i n} C_{i n}-F_{1-2} C_{1}-\beta_{1} U \\
& \varepsilon V_{2} \frac{d C_{2}}{d t}=F_{1-2} C_{1}-F_{d} C_{2}-\beta_{2} U
\end{aligned}
$$

where

$V_{1}, V_{2} \quad$ volumes of top and bottom layer

$F_{i n}, F_{1-2}, F_{d}$ flow rates in, between layer 1 and 2 , and towards the drain

$C_{1}, C_{2} \quad$ concentrations in top and bottom layer
$U$

$\beta_{1}, \beta_{2} \quad$ distribution of the uptake over the two layers, $\beta_{1}+\beta_{2}=1$.

The model applies to a time scale that is large with respect to the irrigation cycles. The parameter $\varepsilon$ is then a calibration parameter $(<1)$ to account for the water retention and the effective mixing volume.

If the drain flow is controlled, the control action of the water controller on a large time scale $\left(F_{i n}\right)$ can be deduced from the overall water balance, whereas $F_{1-2}$ can be found from the water balance over the first layer

$$
\begin{aligned}
& 0=F_{\text {in }}-F_{1-2}-\alpha_{1} W \\
& 0=F_{\text {in }}-F_{d}-W
\end{aligned}
$$

where $W$ is the water uptake rate, and $\alpha_{1}<1$ expresses the fraction of water uptake in the top compartment. Introducing the notation $x=\left[C_{1} C_{2}\right]^{T}, u_{w}=F_{i n}, u_{n}=C_{i n}$, $d_{w}=W, d_{n}=U, p=\varepsilon\left[\begin{array}{ll}V_{1} & V_{2}\end{array}\right]$, where $x, u$, and $d$ are states, controls and disturbances, respectively, and using a drain flow setpoint $y_{w s p}=F_{d}$ leads to

$$
\begin{aligned}
& p_{1} \dot{x}_{1}=u_{w} u_{n}-\left(y_{w s p}+d_{w}-\alpha_{1} d_{w}\right) x_{1}-\beta_{1} d_{n} \\
& p_{2} \dot{x}_{2}=\left(y_{w s p}+d_{w}-\alpha_{1} d_{w}\right) x_{1}-y_{w s p} x_{2}-\beta_{2} d_{n}
\end{aligned}
$$

Hence, when all water uptake takes place in the top segment, this is a linear system, with a system matrix that is a time invariant function of the known drain set point. This is the limit case for the more general situation where the water uptake is distributed. Then, the system is still linear, but time variant in function of the water uptake. Note that the water controller action $u_{w}$ is known. If the top segment represents the plant pot placed on top of the substrate, its volume is small with respect to the total volume, and the system degenerates to a singular perturbed system, leading to

$$
p_{2} \dot{x}_{2}=u_{w} u_{n}-y_{w s p} x_{2}-d_{n}
$$

which is a time invariant (first order) linear system, even when $\alpha_{1}$ is not one. This is another limit case.

Equation (4) shows that the time constant ( $\left.p_{2} / y_{w s p}\right)$ depends upon the set drain flow. Also, the equation shows that the control input needs to be adjusted for the irrigation flow resulting from the water controller, thus creating an natural way to adapt to variations induced by the strong daily evaporation cycle. If desired, with equation (3) a relation can be made with the concentration in the top layer, which may be more relevant to the crop than the drain itself.

In designing the controller, a certain degree of time variance in response to the evapo-transpiration of the crop needs to be considered, depending upon the expected water distribution. The 2-D model of Heinen can be used to test this. 


\subsection{Comparing model to experiments}

According to the analysis above, in a flow-controlled situation, we expect rather simple second or first order models to describe the nutrient dynamics on a time scale relevant to the crop. The results from the experiments support this overall picture. Based on equation (4) a time constant is expected equal to $\varepsilon V / F_{d}$ where $V$ is somewhere between the volume of the rockwool slab $\left(V_{2}\right)$ and the volume of slab plus plant pot $\left(V_{1}+V_{2}\right)$. Using the volume information and an average water retention between 70 and $75 \%$, the expected residence time would be between 5000 and 6000 minutes in the $11 \mathrm{~h}^{-1}$ case, and between 1700 and $2000 \mathrm{l} \mathrm{h}^{-1}$ when the drain is $3 \mathrm{l} \mathrm{h}^{-1}$. In view of the uncertainty in true water retention this compares well with the observed values.

\section{CONCLUSION}

The current accuracy of the data acquisition system does not allow more elaborate models than a first order model with dead time. The time constant is in the order of 5500 minutes for the current measurement gully. No precise statements on the dead time can be made. Not only the processing time of the analyser ( 15 minutes) is important, but also the residence time in the collection vessels. It is probably save to set the total dead time equal to $100-200$ minutes. There is some tendency for variation of time constants over step up and down, although the data are not fully decisive. The design must cope with parameter uncertainty as indicated.

The preliminary result of the increased flow rate experiment shows that the time constant is roughly inversely proportional to the drain flow rate, as expected. This fact must be taken into account in the controller design, so that the controller parameters are adjusted automatically when the user changes the drain flow rate set point. Also, in a situation with plants, the theoretical analysis suggests that the control input needs to be adjusted for the irrigation flow needed to keep the integrated drain volume per time unit constant.

Despite the inherent complexity due to the pulse-wise water supply, the nutrient dynamics on the relevant larger time scale can be described by a simple first order system plus delay. This is a significant finding on the way to arrive at commercial controllers.

\section{REFERENCES}

LeBot, J., S. Adamowicz and P. Robin (1998). Modelling plant nutrition of horticultural crops: a review. Scientia Horticulturae, 74, 47-82.
Dorais, M., A.P. Papadopoulos and A. Gosselin (2001). Influence of electric conductivity management on greenhouse tomato yield and fruit quality. Agronomie, 21, 367-383.

Heinen, M. (2001). FUSSIM2: brief description of the simulation model and application to fertigation scenarios. Agronomie, 21, 285-296.

Kläring, H.-P. (2001). Strategies to control water and nutrient supplies to greenhouse crops. A review. Agronomie, 21, 311-321.

van Straten, G. and Th. H. Gieling (2004). Ion control in closed growing systems with inert media: controller settings and modes of operation. Agricultural Engineering International: the CIGR Journal of Scientific Research and Development (CIGR e-Journal). VI. Paper BC 03 016, 14 pp.

Gieling ,Th.H., H.J.J. Janssen, H.J.J., G. van Straten, M. Suurmond (200). Identification and simulated control of greenhouse closed water supply systems. Computers and Electronics in Agriculture, 26, 361-374.

De Rijck G. and E. Schrevens (1995). Application of mixture-theory for the optimisation of the composition of the nutrient solution. Acta Horticulturae, 401, 283-291.

\section{ACKNOWLEDGEMENT}

Part of this research was funded by the EET agency of the Ministry of Economic Affairs under grant no. EETK99038, in cooperation with Hortimax BV, Pijnacker; PRIVA BV, De Lier; Hydrion BV, Wageningen; Alterra and Plant Research International of Wageningen University and Research Centre. 